\title{
Molecular Mechanics Energy Calculations for the Double-Stranded Helices of Isotactic Poly(methyl methacrylate) Large Oligomers
}

\author{
Hiroshi Kusanagi, Yozo Chatani,* and Hiroyuki Tadokoro* \\ Unitika Research Laboratories Inc., Uji, Kyoto 611, Japan \\ * Department of Macromolecular Science, Faculty of Science, Osaka University, \\ Toyonaka, Osaka 560, Japan
}

(Received May 1, 1996)

\begin{abstract}
For isotactic poly(methyl methacrylate), molecular mechanics energy calculations were carried out on several double-stranded helix oligomer models for analyzing the energetical stability of the polymer double-stranded helix determined by X-ray analysis. The calculation results indicate that the double-stranded-helix structures can exist as stable double-stranded helices by the favorable intertwined interactions of the van der Waals interactions for the large oligomers $(n \geqq 10 ; n$ is the number of monomers in the oligomer). Moreover, the calculated double-stranded helix structure by the energy minimization method is in good agreement with the observed structure by X-ray analysis.

KEY WORDS Energy Calculation / Molecular Mechanics / Double-Stranded Helix / Poly(methyl methacrylate) / Stabilizing Energy /
\end{abstract}

Isotactic poly(methyl methacrylate) (it-PMMA) has known to take the $(10 / 1)$ double-stranded helix in the crystal. ${ }^{1}$ It would seem to be important to clarify the structural reasons why it-PMMA does form the doublestranded helix, although there is no strong interactions between the two chains in the double-stranded helix, such as, for example, hydrogen bonding in the doublestranded helices of $\mathrm{DNA}^{2,3}$ and PEI. ${ }^{4}$ In a previous paper, $a b$ initio molecular orbital (MO) calculations were made to evaluate the energetical stability of the double-stranded monomer model of it-PMMA as a model compound for polymer double-stranded helix. ${ }^{5}$ And the monomer model compound was found to be stabilized with the van der Waals interactions. That is, when the 4-31G basis set and the SCF-MO method with the 2nd order Moeller-Plesset (MP2) perturbation theory were adopted, the calculated total MO energy of the double-stranded monomer model gave the stabilizing energy $(=$ the molecular complex formation energy) of $\Delta E=-0.26 \mathrm{kcal} \mathrm{mol}^{-1}$ of monomer, in comparison with twice of the isolated monomer. And the $a b$ initio MO method reveals a strong theoretical proof that the double-stranded helix structure of it-PMMA is correct. But, this monomer model is too small and not enough to consider polymer model, because it is neglect in this case that the polymer effect, i.e., the long range intermolecular interaction energies along the helix axis between the intertwined two chains, may contribute to the increasing in this stabilizing energy. That is, the ab initio MO method has a serious limitation on the number of involving atoms for the calculation on appropriate large oligomer models. In the viewpoint of polymer stereochemistry, however, the molecular-weight $\left(M_{w}\right)$ dependency of the structural stability of polymer helix is an important research theme. Therefore, in the present study we aimed the energy calculation for oligomer models with rather large molecular-weight by using the molecular mechanics (MM) method, which can be applicable to their energy calculations.

\section{METHODS}

For it-PMMA, nine kinds of oligomer models of $\mathrm{H}-\left[\mathrm{C}\left(\mathrm{COOCH}_{3}\right)\left(\mathrm{CH}_{3}\right)-\mathrm{CH}_{2}\right]_{n}-\mathrm{H}: n=1,2,3,4,5,10$, $15,20,25$, which give the maximum $M_{w}=2502$ at $n=25$, were constructed by adopting the structural parameters determined by X-ray analysis. ${ }^{1}$ Their double-stranded helical molecular models are shown in Figure 1.

MM energy calculations were carried out by using the program of the packing energy minimization for polymers and their potential parameters. ${ }^{6}$ For the electrostatic interactions, point charges are adopted to ester groups and parameter set were used as the same and similar chemical groups. ${ }^{7,8}$ Kubota RS3230 computer work station was used for the calculation.

\section{RESULTS AND DISCUSSION}

\section{Molecular-Weight Dependency of the Stabilizing Energy}

Table I summarizes the intermolecular interaction energy between the two single helical chains of the double-stranded helix model oligomers of it-PMMA determined by $\mathrm{X}$-ray analysis. The negative value of energy shows the attractive force acting between the two chains. The van der Waals and electrostatic interactions gave the attractive and repulsive forces, respectively, for all oligomer models. Values of the total intermolecular interaction energy $E_{\text {Inter }}$ were positive from monomer to trimer models, and beyond trimer became negative. Therefore, the double-stranded helix models of some small oligomers are unstable, while the double-stranded helix models of large oligomers are stable. In order to estimate exactly the energetical stability of the doublestranded helix oligomer models, the normalized stabilizing energy per monomeric unit, $\Delta E$, is defined by $\Delta E=$ $E_{\text {Inter }} / n$, where $n$ is the number of monomers of the oligomer, and the values of $\Delta E$ were listed in Table II. In Figure 2, $\Delta E$ was plotted against the value of $n$. The molecular-weight dependency of the normalized stabilizing energy $\Delta E$ for oligomers reveals some insights.

At first, it was found that with the increasing in number 

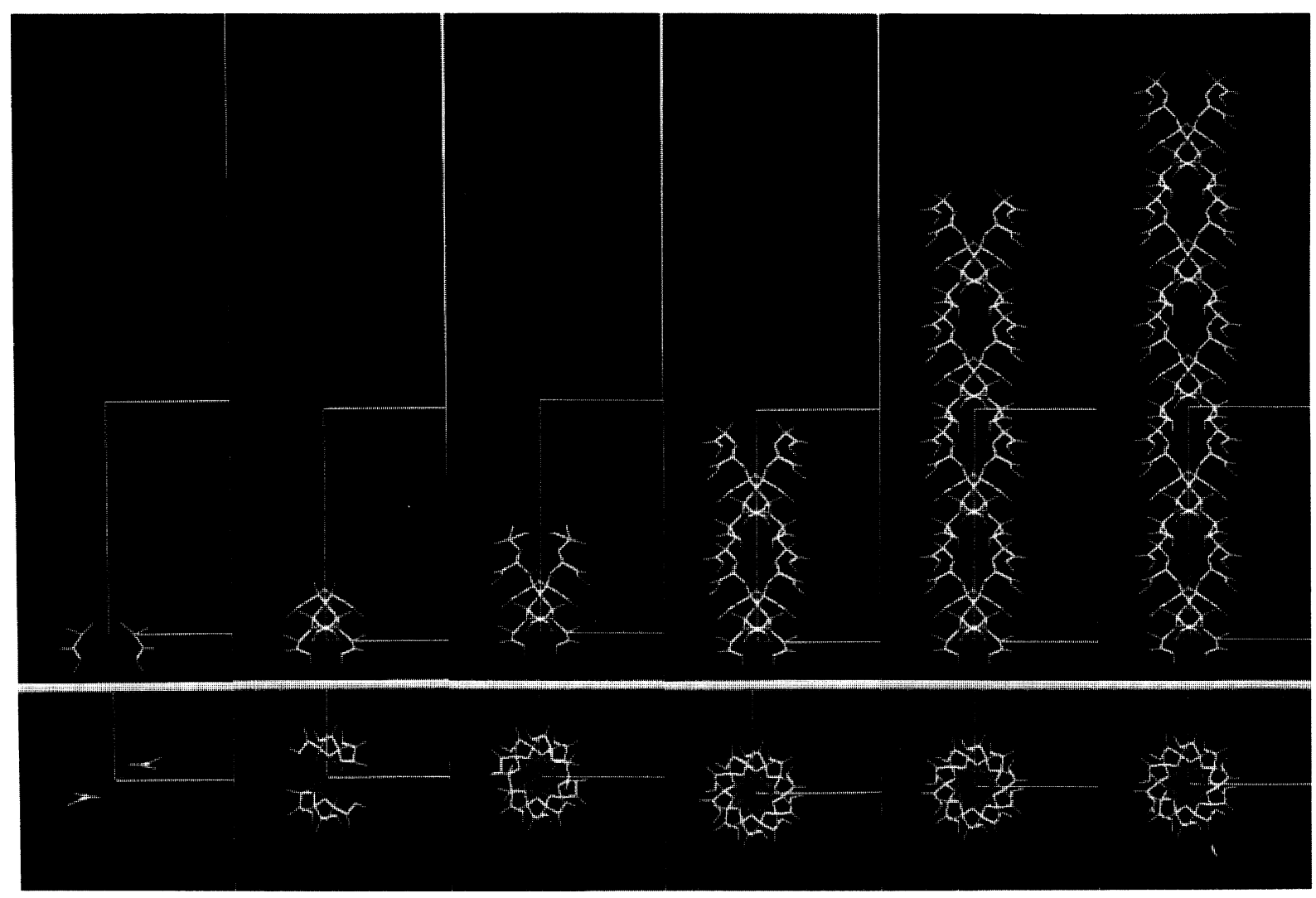

(a) (b)

(c)

(d)

(e)

(f)

Figure 1. Double-stranded helix models of it-PMMA oligomers. (a) monomer, (b) trimer, (c) pentamer, (d) 10-monomer, (e) 20-monomer, and (f) 25-monomer.

Table I. Intermolecular interaction energies between the two single helical chains of the double-stranded helix model oligomers of it-PMMA determined by X-ray analysis

\begin{tabular}{|c|c|c|c|c|c|c|c|c|c|}
\hline $\begin{array}{l}\text { Monomer No. } \\
\text { in oligomer }\end{array}$ & 1 & 2 & 3 & 4 & 5 & 10 & 15 & 20 & 25 \\
\hline$E_{\mathrm{vdw}}$ & -0.5 & -3.2 & -7.0 & -12.1 & -17.4 & -44.5 & -71.5 & -98.5 & -125.5 \\
\hline$E_{\mathrm{el}}$ & 3.5 & 6.2 & 8.0 & 9.2 & 10.1 & 14.3 & 18.5 & 22.6 & 26.8 \\
\hline$E_{\text {Inter }}^{\mathrm{a}}$ & 3.0 & 3.0 & 1.0 & -2.9 & -8.9 & -30.2 & -53.0 & -75.9 & -98.7 \\
\hline
\end{tabular}

${ }^{\mathrm{a}}$ Intermolecular interaction energy $E_{\mathrm{Inter}}: E_{\mathrm{Inter}}=E_{\mathrm{vdW}}+E_{\mathrm{el}}\left(\mathrm{kcal} \mathrm{mol}{ }^{-1}\right.$ of oligomer), where $E_{\mathrm{vdw}}$ and $E_{\mathrm{el}}$ are the van der Waals and electrostatic energies, respectively.

Table II. Stabilizing energies $\Delta E$ for double-stranded helix model oligomers of it-PMMA structure determined by X-Ray analysis

\begin{tabular}{|c|c|c|c|c|c|c|c|c|c|}
\hline $\begin{array}{l}\text { Monomer No. } \\
\text { in oligomer }\end{array}$ & 1 & 2 & 3 & 4 & 5 & 10 & 15 & 20 & 25 \\
\hline$\Delta E_{\mathrm{vdw}}$ & -0.5 & -1.6 & -2.3 & -3.1 & -3.5 & -4.5 & -4.8 & -4.9 & -5.0 \\
\hline$\Delta E_{\mathrm{el}}$ & 3.5 & 3.1 & 2.7 & 2.4 & 2.0 & 1.4 & 1.2 & 1.1 & 1.0 \\
\hline$\Delta E^{\mathrm{a}}$ & 3.0 & 1.5 & 0.4 & -0.7 & -1.5 & -3.1 & -3.6 & -3.8 & -4.0 \\
\hline
\end{tabular}

${ }^{\text {a }}$ Stabilizing energy $\Delta E: \Delta E\left(\mathrm{kcal} \mathrm{mol}^{-1}\right.$ of monomer $)=E_{\text {Inter }} / n$, where $n$ is the number of monomer in the oligomer; $\Delta E=\Delta E_{\mathrm{vdw}}+\Delta E_{\mathrm{el}}$, where $\Delta E_{\mathrm{vdw}}$ and $\Delta E_{\mathrm{el}}$ are the van der Waals and electrostatic energies, respectively.

of $n$, the stabilizing energy $\Delta E$ increases rapidly at $n=1-10$, and becomes almost to a saturate value of $\Delta E=-4.0 \mathrm{kcal} \mathrm{mol}^{-1}$ at $n=20-25$. That is, the expected large molecular-weight effect for the stabilizing energy has been confirmed in the present study. Therefore, one of our initial purposes has been achieved. On the other hand, for the double-stranded helix of it-PMMA monomer, the present calculation result of stabilizing energy $\left(\Delta E=3.0 \mathrm{kcal} \mathrm{mol}^{-1}\right.$ : repulsive energy) is in disagreement with the calculation $(\Delta E=-0.26$ $\mathrm{kcal} \mathrm{mol}^{-1}$ : attractive energy) by the ab initio $\mathrm{MO}$ method in the previous paper. ${ }^{5}$ In this case, the origin of the disagreement must attribute to the MM energy calculation method. If we will introduce a scaling factor between the van der Waals and electrostatic interactions, this disagreement may be apparently solved. The MM energy calculation method has a problem with respect to the scaling among the energy components when the intra- 
and intermolecular interactions are divided to the bondlength deformation energy, bond-angle deformation energy, torsion-angle deformation energy, van der Waals energy, electrostatic energy, and hydrogen-bond energy and so on. Therefore, we are now investigating to develop a procedure that can calculate the large oligomer by using the $a b$ initio MO method. However, this does not mean the un-usefulness of the MM method. For an example, a systematic change of the stabilizing energy $\Delta E$ against $n$ is an utilized information for analyzing the structural

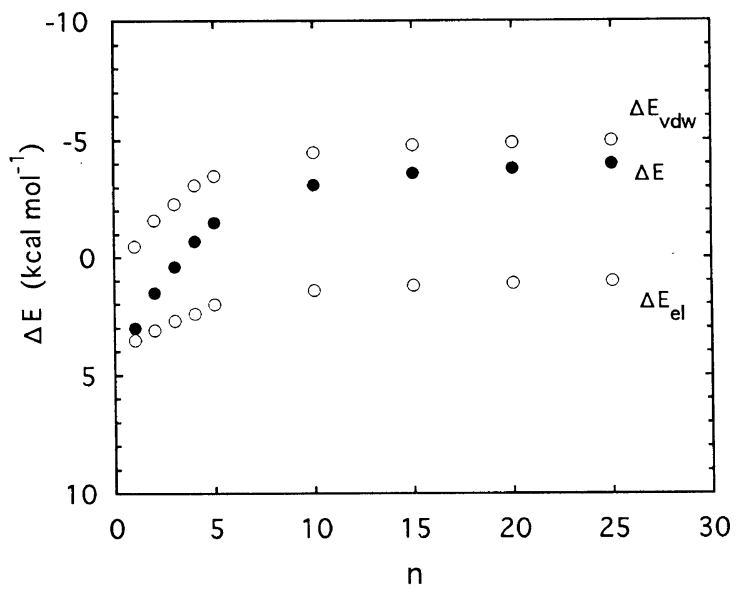

Figure 2. Molecular weight dependency of stabilizing energy $\Delta E_{\mathrm{stb}}$. for double-stranded helix models of it-PMMA oligomers. stability of double-stranded helix of it-PMMA.

Secondary, the calculation results suggest that small oligomers might have non-double-stranded helix structure, and the critical number of $n$ for the formation of double-stranded helix structure is about 10 from the curvature of $\Delta E$ as shwon in Figure 2. The single chain of the oligomer with $n=10$ forms just one turn of helix (see Figure 1), thereby the stabilization of their doublestranded helix seems to be almost completed. Therefore, this value $n=10$ may be a magic number for the stabilizing of the double-stranded helix of it-PMMA, and the stabilization of the double-stranded helix may closely relate the $(10 / 1)$ helical symmetry of it-PMMA; $(10 / 1)$ means the helix with 10 monomeric units per one turn.

\section{Comparison between the X-Ray and Energy Calculation Results}

The energy minimization for total energy $E_{\text {Total }}$ of intra-and intermolecular interactions, $E_{\text {Intra }}$ and $E_{\text {Inter }}$, of the double stranded helix oligomer models were carried out with the structure obtained by the X-ray analysis. Internal rotation angles of main- and side-chain and also bond angles of main-chain were taken as variable parameters. Table III lists the total number of parameters and the energy values after the energy optimization with the packing energy minimization method, in comparison with these of the initial structures by $\mathrm{X}$ ray analysis. The normalized stabilizing energies $\Delta E$

Table III. Energy minimization results for the double-stranded helix oligomer models of it-PMMA structure determined by X-Ray analysis

\begin{tabular}{|c|c|c|c|c|c|c|c|c|c|}
\hline $\begin{array}{l}\text { Monomer No. } \\
\text { in oligomer }\end{array}$ & 1 & 2 & 3 & 4 & 5 & 10 & 15 & 20 & 25 \\
\hline $\begin{array}{c}\text { No. of variable } \\
\text { parameters }\end{array}$ & 4 & 8 & 14 & 20 & 26 & 56 & 86 & 116 & 146 \\
\hline \multicolumn{10}{|c|}{ Intramolecular interaction energies } \\
\hline$E_{\mathrm{vdW}}$ & $\begin{array}{c}3.2 \\
(33.7)^{b}\end{array}$ & $\begin{array}{c}1.6 \\
(4.4)\end{array}$ & $\begin{array}{c}-0.1 \\
(3.1)\end{array}$ & $\begin{array}{r}-0.2 \\
(1.6)\end{array}$ & $\begin{array}{c}-1.4 \\
(0.1)\end{array}$ & $\begin{array}{l}-7.1 \\
(-7.1)\end{array}$ & $\begin{array}{l}-12.2 \\
(-14.4)\end{array}$ & $\begin{array}{l}-16.9 \\
(-21.6)\end{array}$ & $\begin{array}{l}-21.7 \\
(-28.9)\end{array}$ \\
\hline$E_{\mathrm{el}}$ & $\begin{array}{l}1.7 \\
(1.7)\end{array}$ & $\begin{array}{c}4.9 \\
(3.9)\end{array}$ & $\begin{array}{c}6.9 \\
(5.5)\end{array}$ & $\begin{array}{c}7.7 \\
(6.8)\end{array}$ & $\begin{array}{c}8.9 \\
(7.9)\end{array}$ & $\begin{array}{c}14.6 \\
(13.1)\end{array}$ & $\begin{array}{c}20.3 \\
(18.4)\end{array}$ & $\begin{array}{c}26.1 \\
(23.6)\end{array}$ & $\begin{array}{l}31.8 \\
(28.9)\end{array}$ \\
\hline$E_{\Theta}$ & $\begin{array}{c}0.1 \\
(60.4)\end{array}$ & $\begin{array}{c}4.0 \\
(4.5)\end{array}$ & $\begin{array}{c}6.9 \\
(8.9)\end{array}$ & $\begin{array}{c}9.8 \\
(13.4)\end{array}$ & $\begin{array}{c}13.2 \\
(17.9)\end{array}$ & $\begin{array}{c}31.9 \\
(40.2)\end{array}$ & $\begin{array}{c}52.0 \\
(62.6)\end{array}$ & $\begin{array}{c}71.7 \\
(84.9)\end{array}$ & $\begin{array}{c}91.3 \\
(107.3)\end{array}$ \\
\hline$E_{\tau}$ & $\begin{array}{c}0.4 \\
(2.1)\end{array}$ & $\begin{array}{c}0.6 \\
(3.1)\end{array}$ & $\begin{array}{c}1.4 \\
(4.0)\end{array}$ & $\begin{array}{c}2.6 \\
(4.9)\end{array}$ & $\begin{array}{c}3.5 \\
(5.8)\end{array}$ & $\begin{array}{c}6.9 \\
(10.4)\end{array}$ & $\begin{array}{l}10.3 \\
(14.9)\end{array}$ & $\begin{array}{c}13.7 \\
(19.5)\end{array}$ & $\begin{array}{c}17.1 \\
(24.0)\end{array}$ \\
\hline$E_{\text {Intra }}$ & $\begin{array}{c}5.4 \\
(97.9)\end{array}$ & $\begin{array}{c}11.1 \\
(83.4)\end{array}$ & $\begin{array}{c}15.1 \\
(21.5)\end{array}$ & $\begin{array}{c}19.9 \\
(26.7)\end{array}$ & $\begin{array}{c}24.2 \\
(31.7)\end{array}$ & $\begin{array}{c}46.3 \\
(56.6)\end{array}$ & $\begin{array}{c}70.4 \\
(81.5)\end{array}$ & $\begin{array}{c}94.3 \\
(106.4)\end{array}$ & $\begin{array}{c}118.5 \\
(138.5)\end{array}$ \\
\hline \multicolumn{10}{|c|}{ Intermolecular interactin energies } \\
\hline$E_{\mathrm{vdW}}$ & $\begin{array}{l}-0.3 \\
(-0.5)\end{array}$ & $\begin{array}{c}-3.0 \\
(-3.2)\end{array}$ & $\begin{array}{c}-6.1 \\
(-7.0)\end{array}$ & $\begin{array}{c}-12.5 \\
(-12.1)\end{array}$ & $\begin{array}{c}-18.4 \\
(-17.4)\end{array}$ & $\begin{array}{c}-47.7 \\
(-44.5)\end{array}$ & $\begin{array}{c}-78.2 \\
(-71.5)\end{array}$ & $\begin{array}{l}-108.6 \\
(-98.5)\end{array}$ & $\begin{array}{c}-139.1 \\
(-125.5)\end{array}$ \\
\hline$E_{\mathrm{el}}$ & $\begin{array}{c}3.3 \\
(3.5)\end{array}$ & $\begin{array}{c}5.6 \\
(6.2)\end{array}$ & $\begin{array}{c}7.1 \\
(8.0)\end{array}$ & $\begin{array}{c}8.6 \\
(9.2)\end{array}$ & $\begin{array}{c}9.5 \\
(10.1)\end{array}$ & $\begin{array}{c}13.3 \\
(14.3)\end{array}$ & $\begin{array}{c}17.4 \\
(18.5)\end{array}$ & $\begin{array}{c}21.5 \\
(22.6)\end{array}$ & $\begin{array}{c}25.6 \\
(26.8)\end{array}$ \\
\hline$E_{\text {Inter }}$ & $\begin{array}{c}3.0 \\
(3.0)\end{array}$ & $\begin{array}{c}2.7 \\
(3.0)\end{array}$ & $\begin{array}{c}1.0 \\
(1.0)\end{array}$ & $\begin{array}{c}-3.8 \\
(-2.9)\end{array}$ & $\begin{array}{c}-8.9 \\
(-8.9)\end{array}$ & $\begin{array}{l}-34.3 \\
(-30.2)\end{array}$ & $\begin{array}{c}-60.8 \\
(-53.0)\end{array}$ & $\begin{array}{c}-87.1 \\
(-75.9)\end{array}$ & $\begin{array}{l}-113.5 \\
(-98.7)\end{array}$ \\
\hline \multicolumn{10}{|c|}{ Total energy ${ }^{\mathrm{a}}\left(\mathrm{kcal} \mathrm{mol}^{-1}\right)$} \\
\hline$E_{\text {Total }}$ & $\begin{array}{c}8.4 \\
(100.9)\end{array}$ & $\begin{array}{c}13.8 \\
(86.4)\end{array}$ & $\begin{array}{c}16.1 \\
(22.5)\end{array}$ & $\begin{array}{c}16.1 \\
(23.8)\end{array}$ & $\begin{array}{c}15.3 \\
(22.8)\end{array}$ & $\begin{array}{c}12.0 \\
(26.4)\end{array}$ & $\begin{array}{c}9.6 \\
(28.5)\end{array}$ & $\begin{array}{c}7.2 \\
(30.5)\end{array}$ & $\begin{array}{c}5.0 \\
(39.8)\end{array}$ \\
\hline
\end{tabular}

${ }^{\mathrm{a}} E_{\text {Total }}=E_{\text {Intra }}+E_{\text {Inter }} ; E_{\text {Intra }}=E_{\mathrm{vdW}}+E_{\mathrm{el}}+E_{\Theta}+E_{\tau} ; E_{\mathrm{Inter}}=E_{\mathrm{vdW}}+E_{\mathrm{el}}$, where $E_{\mathrm{vdw}}, E_{\mathrm{el}}, E_{\boldsymbol{\Theta}}$, and $E_{\tau}$ are the van der Waals, electrostatic, bond-angle defoermation, and torsion-angle deformation energies, respectively. ${ }^{\mathrm{b}}($ ) indicates the energy value of the structure by X-ray analysis. 
Table IV. Stabilizing energies $\Delta E$ for the energy minimized double-stranded helix model oligomers

\begin{tabular}{|c|c|c|c|c|c|c|c|c|c|}
\hline $\begin{array}{l}\text { Monomer No. } \\
\text { in oligomer }\end{array}$ & 1 & 2 & 3 & 4 & 5 & 10 & 15 & 20 & 25 \\
\hline$\Delta E_{\mathrm{vdW}}$ & $\begin{array}{c}-0.3 \\
(-0.3)^{\mathrm{b}}\end{array}$ & $\begin{array}{l}-1.5 \\
(-1.5)\end{array}$ & $\begin{array}{c}-2.0 \\
(-2.3)\end{array}$ & $\begin{array}{c}-3.1 \\
(-3.1)\end{array}$ & $\begin{array}{c}-3.7 \\
(-3.5)\end{array}$ & $\begin{array}{c}-4.8 \\
(-4.5)\end{array}$ & $\begin{array}{c}-5.2 \\
(-4.8)\end{array}$ & $\begin{array}{c}-5.4 \\
(-4.9)\end{array}$ & $\begin{array}{c}-5.5 \\
(-5.0)\end{array}$ \\
\hline$\Delta E_{\mathrm{el}}$ & $\begin{array}{c}3.3 \\
(3.3)\end{array}$ & $\begin{array}{c}2.8 \\
(3.1)\end{array}$ & $\begin{array}{c}2.4 \\
(2.7)\end{array}$ & $\begin{array}{c}2.2 \\
(2.4)\end{array}$ & $\begin{array}{c}1.9 \\
(2.0)\end{array}$ & $\begin{array}{c}1.3 \\
(1.4)\end{array}$ & $\begin{array}{c}1.2 \\
(1.2)\end{array}$ & $\begin{array}{c}1.1 \\
(1.1)\end{array}$ & $\begin{array}{c}1.0 \\
(1.0)\end{array}$ \\
\hline$\Delta E^{\mathrm{a}}$ & $\begin{array}{c}3.0 \\
(3.0)\end{array}$ & $\begin{array}{c}1.3 \\
(1.5)\end{array}$ & $\begin{array}{c}0.4 \\
(0.4)\end{array}$ & $\begin{array}{c}-0.9 \\
(-0.7)\end{array}$ & $\begin{array}{l}-1.8 \\
(-1.5)\end{array}$ & $\begin{array}{l}-3.5 \\
(-3.1)\end{array}$ & $\begin{array}{c}-4.0 \\
(-3.6)\end{array}$ & $\begin{array}{l}-4.3 \\
(-3.8)\end{array}$ & $\begin{array}{c}-4.5 \\
(-4.0)\end{array}$ \\
\hline
\end{tabular}

${ }^{\mathrm{a}}$ Stabilizing energy $\Delta E: \Delta E\left(\mathrm{kcal} \mathrm{mol}{ }^{-1}\right.$ of monomer $)=E_{\mathrm{Inter}} / n$, where $n$ is the number of monomer in the oligomer; $\Delta E=\Delta E_{\mathrm{vdw}}+\Delta E_{\mathrm{el}}$. ${ }^{\mathrm{b}}(\quad)$ indicates the energy value of the structure by X-ray analysis.

$\operatorname{mgbcx}$
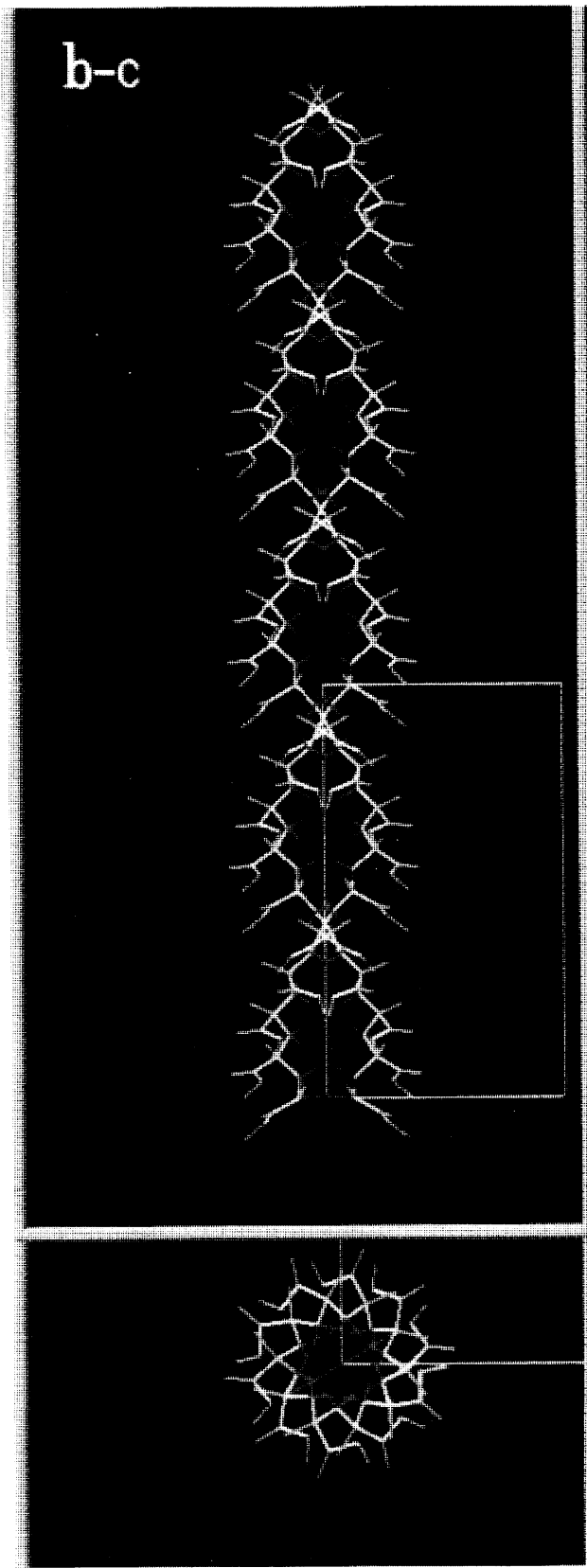

(a) $m g b c x$
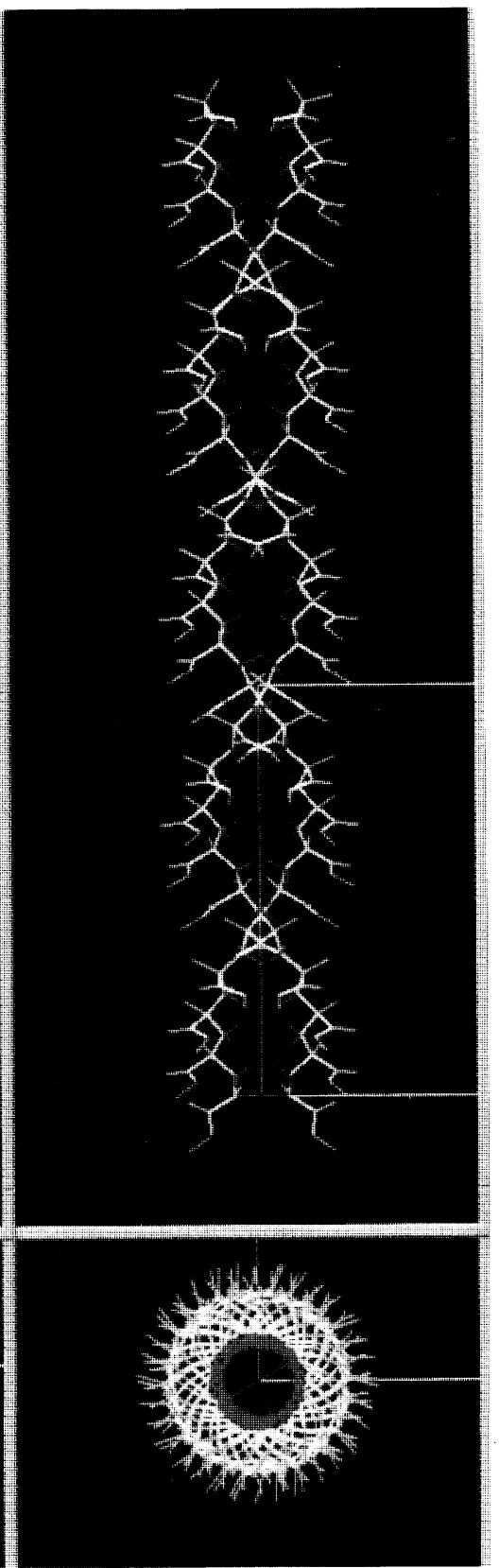

(b)

Figure 3. Comparison between the double-stranded helix oligomer models with 25 -monomer by the X-ray analysis and energy calculation procedure. (a) Before and (b) after energy minimization models. 
were also listed in Table IV. After the energy minimization, total energy $E_{\text {Total }}$ showed a large reduction but the variation of intermolecular interaction energy $E_{\text {Inter }}$ is small. This reveals that the double-stranded helix structure determined by X-ray analysis is well stabilized by the intermolecular interactions. For the oligomer with
25 monomers, the double-stranded helix models before and after energy minimization are shown in Figure 3. The variation of bond angles and internal rotation angle during the energy minimization is small (Table V), especially the observed large deviations of the bond angles from the standard value (tetrahedral angle

Table V. Variations of bond angle and internal rotation angles during the energy minimization of double-stranded helix of 25 -monomer

\begin{tabular}{|c|c|c|c|c|c|c|c|c|}
\hline \multicolumn{3}{|c|}{ Bond angle $^{a}$} & \multicolumn{6}{|c|}{ Rotational angle ${ }^{a}$} \\
\hline & Initial & Final & & Initial & Final & & Initial & Final \\
\hline$\theta_{1 \mathrm{a}}$ & 124.0 & 121.9 & $\tau_{1 \mathrm{a}}$ & 198.2 & 199.0 & $\tau_{13 \mathrm{~b}}$ & 197.4 & 194.8 \\
\hline$\theta_{1 \mathrm{~b}}$ & 103.6 & 103.2 & $\tau_{1 \mathrm{~b}}$ & 197.4 & 196.4 & $\tau_{13 \mathrm{c}}$ & 63.0 & 59.6 \\
\hline$\theta_{2 \mathrm{a}}$ & 124.0 & 122.6 & $\tau_{1 \mathrm{c}}$ & 63.0 & 57.8 & $\tau_{13 \mathrm{~d}}$ & 190.0 & 179.3 \\
\hline$\theta_{2 \mathrm{~b}}$ & 103.6 & 103.2 & $\tau_{1 \mathrm{~d}}$ & 190.0 & 185.4 & $\tau_{14 \mathrm{a}}$ & 198.2 & 199.7 \\
\hline$\theta_{3 \mathrm{a}}$ & 124.0 & 122.9 & $\tau_{2 \mathrm{a}}$ & 198.2 & 196.1 & $\tau_{14 \mathrm{~b}}$ & 197.4 & 194.8 \\
\hline$\theta_{3 b}$ & 103.6 & 103.6 & $\tau_{2 \mathrm{~b}}$ & 197.4 & 196.0 & $\tau_{14 \mathrm{c}}^{14 \mathrm{~b}}$ & 63.0 & 59.5 \\
\hline$\theta_{4 a}$ & 124.0 & 122.9 & $\tau_{2 \mathrm{c}}$ & 63.0 & 57.9 & $\tau_{14 \mathrm{~d}}$ & 190.0 & 179.3 \\
\hline$\theta_{4 \mathrm{~b}}$ & 103.6 & 103.6 & $\tau_{2 \mathrm{~d}}$ & 190.0 & 185.4 & $\tau_{15 \mathrm{a}}$ & 198.2 & 199.8 \\
\hline$\theta_{5 \mathrm{a}}$ & 124.0 & 122.6 & $\tau_{3 \mathrm{a}}$ & 198.2 & 196.7 & $\tau_{15 \mathrm{~b}}$ & 197.4 & 194.8 \\
\hline$\theta_{5 \mathrm{~b}}$ & 103.6 & 103.6 & $\tau_{3 \mathrm{~b}}$ & 197.4 & 197.7 & $\tau_{15 \mathrm{c}}$ & 63.0 & 59.5 \\
\hline$\theta_{6 a}$ & 124.0 & 122.9 & $\tau_{3 \mathrm{c}}$ & 63.0 & 63.4 & $\tau_{15 \mathrm{~d}}$ & 190.0 & 179.3 \\
\hline$\theta_{6 \mathrm{~b}}$ & 103.6 & 103.6 & $\tau_{3 \mathrm{~d}}$ & 190.0 & 180.6 & $\tau_{16 \mathrm{a}}$ & 198.2 & 199.8 \\
\hline$\theta_{7 \mathrm{a}}$ & 124.0 & 123.0 & $\tau_{4 \mathrm{a}}$ & 198.2 & 200.0 & $\tau_{16 \mathrm{~b}}$ & 197.4 & 194.8 \\
\hline$\theta_{7 \mathrm{~b}}$ & 103.6 & 103.8 & $\tau_{4 \mathrm{~b}}$ & 197.4 & 197.5 & $\tau_{16 \mathrm{c}}$ & 63.0 & 59.5 \\
\hline$\theta_{8 \mathrm{a}}$ & 124.0 & 123.1 & $\tau_{4 \mathrm{c}}$ & 63.0 & 61.3 & $\tau_{16 \mathrm{~d}}$ & 190.0 & 179.3 \\
\hline$\theta_{8 \mathrm{~b}}$ & 103.6 & 103.9 & $\tau_{4 \mathrm{~d}}$ & 190.0 & 181.7 & $\tau_{17 \mathrm{a}}$ & 198.2 & 199.8 \\
\hline$\theta_{9 \mathrm{a}}$ & 124.0 & 123.1 & $\tau_{5 \mathbf{a}}$ & 198.2 & 200.9 & $\tau_{17 \mathrm{~b}}$ & 197.4 & 194.8 \\
\hline$\theta_{9 \mathrm{~b}}$ & 103.6 & 103.9 & $\tau_{5 b \mathrm{~b}}$ & 197.4 & 196.6 & $\tau_{17 \mathrm{c}}$ & 63.0 & 59.5 \\
\hline $0_{10 \mathrm{a}}$ & 124.0 & 123.1 & $\tau_{5 \mathrm{c}}$ & 63.0 & 59.2 & $\tau_{17 \mathrm{~d}}$ & 190.0 & 179.3 \\
\hline$\theta_{10 \mathrm{~b}}$ & 103.6 & 103.9 & $\tau_{5 d}$ & 190.0 & 181.5 & $\tau_{18 \mathrm{a}}$ & 198.2 & 199.8 \\
\hline $0_{11 \mathrm{a}}$ & 124.0 & 123.1 & $\tau_{6 \mathrm{a}}$ & 198.2 & 199.7 & $\tau_{18 \mathrm{~b}}$ & 197.4 & 194.9 \\
\hline$\theta_{11 \mathrm{~b}}$ & 103.6 & 104.0 & $\tau_{6 \mathrm{~b}}$ & 197.4 & 195.2 & $\tau_{18 \mathrm{c}}$ & 63.0 & 59.5 \\
\hline $0_{12 \mathrm{a}}$ & 124.0 & 123.1 & $\tau_{6 c}$ & 63.0 & 59.2 & $\tau_{18 \mathrm{~d}}$ & 190.0 & 179.3 \\
\hline$\theta_{12 \mathrm{~b}}$ & 103.6 & 104.0 & $\tau_{6 \mathrm{~d}}$ & 190.0 & 181.8 & $\tau_{19 \mathrm{a}}$ & 198.2 & 199.9 \\
\hline$\theta_{13 \mathrm{a}}$ & 124.0 & 123.1 & $\tau_{7 \mathrm{a}}$ & 198.2 & 199.9 & $\tau_{19 \mathrm{~b}}$ & 197.4 & 195.0 \\
\hline$\theta_{13 \mathrm{~b}}$ & 103.6 & 104.0 & $\tau_{7 \mathrm{~b}}$ & 197.4 & 195.2 & $\tau_{19 \mathrm{c}}$ & 63.0 & 59.4 \\
\hline$\theta_{14 \mathrm{a}}$ & 124.0 & 123.1 & $\tau_{7 \mathrm{c}}$ & 63.0 & 59.5 & $\tau_{19 \mathrm{~d}}$ & 190.0 & 179.3 \\
\hline$\theta_{14 \mathrm{~b}}$ & 103.6 & 104.0 & $\tau_{7 \mathrm{~d}}$ & 190.0 & 180.1 & $\tau_{20 \mathrm{a}}$ & 198.2 & 200.0 \\
\hline$\theta_{15 \mathrm{a}}$ & 124.0 & 123.1 & $\tau_{8 \mathrm{a}}$ & 198.2 & 199.4 & $\tau_{20 \mathrm{~b}}$ & 197.4 & 195.1 \\
\hline$\theta_{15 \mathrm{~b}}$ & 103.6 & 104.0 & $\tau_{8 \mathrm{~b}}$ & 197.4 & 194.7 & $\tau_{20 \mathrm{c}}$ & 63.0 & 59.3 \\
\hline$\theta_{16 \mathrm{a}}$ & 124.0 & 123.1 & $\tau_{8 \mathrm{c}}$ & 63.0 & 57.8 & $\tau_{20 \mathrm{~d}}$ & 190.0 & 179.4 \\
\hline$\theta_{16 \mathrm{~b}}$ & 103.6 & 104.0 & $\tau_{8 \mathrm{~d}}$ & 190.0 & 185.4 & $\tau_{21 \mathrm{a}}$ & 198.2 & 199.2 \\
\hline$\theta_{17 \mathrm{a}}$ & 124.0 & 123.1 & $\tau_{9 \mathrm{a}}$ & 198.2 & 199.0 & $\tau_{21 \mathrm{~b}}$ & 197.4 & 195.0 \\
\hline$\theta_{17 \mathrm{~b}}$ & 103.6 & 104.0 & $\tau_{9 \mathrm{~b}}$ & 197.4 & 196.4 & $\tau_{21 \mathrm{c}}$ & 63.0 & 59.1 \\
\hline$\theta_{18 \mathrm{a}}$ & 124.0 & 123.0 & $\tau_{9 \mathrm{c}}$ & 63.0 & 59.8 & $\tau_{21 \mathrm{~d}}$ & 190.0 & 179.5 \\
\hline$\theta_{18 \mathrm{~b}}$ & 103.6 & 104.0 & $\tau_{9 \mathrm{~d}}$ & 190.0 & 180.0 & $\tau_{22 \mathrm{a}}$ & 198.2 & 199.8 \\
\hline$\theta_{19 \mathrm{a}}$ & 124.0 & 123.0 & $\tau_{10 \mathrm{a}}$ & 198.2 & 199.5 & $\tau_{22 \mathrm{~b}}$ & 197.4 & 195.4 \\
\hline$\theta_{19 \mathrm{~b}}$ & 103.6 & 104.0 & $\tau_{10 \mathrm{~b}}$ & 197.4 & 195.0 & $\tau_{22 \mathrm{c}}$ & 63.0 & 59.4 \\
\hline$\theta_{20 \mathrm{a}}$ & 124.0 & 122.9 & $\tau_{10 \mathrm{c}}$ & 63.0 & 60.1 & $\tau_{22 \mathrm{~d}}$ & 190.0 & 179.6 \\
\hline$\theta_{20 \mathrm{~b}}$ & 103.6 & 104.0 & $\tau_{10 \mathrm{~d}}$ & 190.0 & 179.4 & $\tau_{23 \mathrm{a}}$ & 198.2 & 199.8 \\
\hline$\theta_{21 \mathrm{a}}$ & 124.0 & 122.9 & $\tau_{11 \mathrm{a}}$ & 198.2 & 199.7 & $\tau_{23 \mathrm{~b}}$ & 197.4 & 198.5 \\
\hline$\theta_{21 \mathrm{~b}}$ & 103.6 & 104.0 & $\tau_{11 \mathrm{~b}}$ & 197.4 & 194.8 & $\tau_{23 \mathrm{c}}$ & 63.0 & 59.6 \\
\hline$\theta_{22 \mathrm{a}}$ & 124.0 & 122.7 & $\tau_{11 \mathrm{c}}$ & 63.0 & 59.6 & $\tau_{23 \mathrm{~d}}$ & 190.0 & 179.5 \\
\hline$\theta_{22 \mathrm{~b}}$ & 103.6 & 104.8 & $\tau_{11 \mathrm{~d}}$ & 190.0 & 179.4 & $\tau_{24 \mathrm{a}}$ & 198.2 & 199.3 \\
\hline$\theta_{23 \mathrm{a}}$ & 124.0 & 121.8 & $\tau_{12 \mathrm{a}}$ & 198.2 & 199.7 & $\tau_{24 \mathrm{~b}}$ & 197.4 & 198.1 \\
\hline$\theta_{23 \mathrm{~b}}$ & 103.6 & 105.2 & $\tau_{12 \mathrm{~b}}$ & 197.4 & 194.8 & $\tau_{24 \mathrm{c}}$ & 63.0 & 59.3 \\
\hline$\theta_{24 \mathrm{a}}$ & 124.0 & 122.0 & $\tau_{12 \mathrm{c}}$ & 63.0 & 59.6 & $\tau_{24 \mathrm{~d}}$ & 190.0 & 180.9 \\
\hline \multirow[t]{2}{*}{$\theta_{24 \mathrm{~b}}$} & 103.6 & 106.7 & $\tau_{12 \mathrm{~d}}$ & 190.0 & 179.3 & $\tau_{25 \mathrm{c}}$ & 63.0 & 63.1 \\
\hline & & & $\tau_{13 \mathrm{a}}$ & 198.2 & 199.7 & $\tau_{25 \mathrm{~d}}$ & 190.0 & 179.2 \\
\hline
\end{tabular}

a The bond angles $\theta$ and internal rotation angles $\tau$ are numbered as follows:

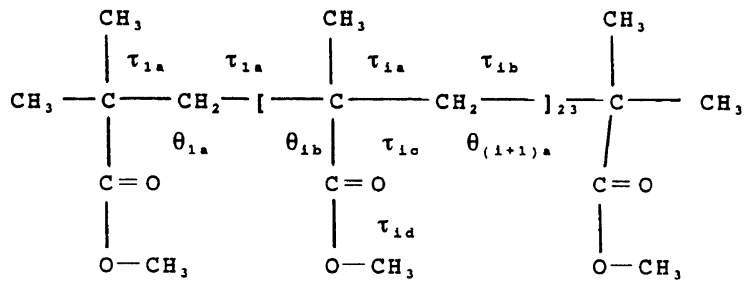


$\theta=109.5^{\circ}$ ) was confirmed by the energy calculations. Energy minimization gave a little loose $(10.6 / 1)(=53 / 5)$ double-stranded helix structure as compared with the (10/1) double-stranded helix of the X-ray model; (53/5) means the helix with 53 monomeric units per 5 turns. The calculated double-stranded helix structures by the energy minimization method are, as a whole, in good agreement with the observed structure by X-ray analysis. Tadokoro and coworkers reported that the stable single helix of it-PMMA takes a fairly loose (12/1) helical symmetry. ${ }^{9}$

Comparing the $\Delta E_{\mathrm{vdW}}$ and $\Delta E_{\mathrm{el}}$ for the energetically stabilized large oligomers $(n \geqq 10)$, the values of $\Delta E_{\mathrm{el}}$ are almost same before and after the energy optimization, while the values of $\Delta E_{\mathrm{vdw}}$ have changed considerably (the maximum $5 \%$ for $n=25$ ) during the geometry optimization. This situation may attribute to the deference in properties of van der Waals (short range) interactions and electrostatic (long range) interactions. That is, the van der Waals interactions is sensitive to the change of secondary structure of polymers, while the electrostatic interactions is insensitive.

\section{CONCLUSION}

Molecular mechanics energy calculations were carried out on several double-stranded helix oligomer models for analyzing the energetically stability of the polymer double-stranded helix structure of it-PMMA determined by $\mathrm{X}$-ray analysis. The calculation results indicate that the double-stranded helix structures can exist as stable double-stranded helices by the favorite intertwined interactions of the van der Waals interactions for the large oligomers $(n \geqq 10)$. The calculated double-stranded helix structure by the energy minimization method is in good agreement with the observed structure by X-ray analysis. This reveals that the double-stranded helix structure of it-PMMA is energetically stable and rather rigid secondary structure by the favorable interhelix van der Waals interactions, although there is a problem for the potential parameters used in the energy calculations.

We think that the energy calculation method is a powerful research procedure if it shall be applied to structural studies, taking into account the probability and limitation on the applicability in the mind.

\section{REFERENCES}

1. H. Kusanagi, Y. Chatani, and H. Tadokoro, Polymer, 35, 2028 (1994).

2. J. D. Watson and H. C. Crick, Nature (London), 171, 737 (1953).

3. R. Lagrige, H. R. Wilson, C. W. Hooper, and M. H. F. Wilkins, J. Mol. Biol., 2, 19 (1960).

4. Y. Chatani, T. Kobatake, H. Tadokoro, and R. Tanaka, Macromolecules, 15, 170 (1982).

5. H. Kusanagi, Polym. J., 28, 639 (1996).

6. H. Kusanagi, Kobunshi Ronbunsyu, 53, 108 (1996)

7. D. R. Ferro, S. Bruckner, S. V. Meille, and M. Ragazzi, Macromolecules, 23, 1676 (1990).

8. S. J. Weiner, P. A. Kollman, D. A. Case, U. C. Singh, C. Chio, G. Alagona, S. Profeta, and P. Weiner, J. Am. Chem. Soc., 106, 765 (1984).

9. H. Tadokoro, K. Tai, M. Yokoyama, and M. Kobayashi, J. Polym. Sci., Polym. Phys. Ed., 11, 825 (1973). 